\title{
PREDICTION OF SCALABLE LOSS IN FRANCIS RUNNERS OF DIFFERENT SPECIFIC SPEED
}

\author{
T.KITAHORA, J.KUROKAWA, M.MATUMOTO \\ Yokohama National University \\ 156 Tokiwadai, Hodogaya-ku, Yokohama 240, Japan \\ R.SUZUKI \\ Fuji Electric Co.,Ltd. \\ 1-1 Tanabeshinden, Kawasaki-ku, Kawasaki 210, Japan
}

\section{Introduction}

Hydraulic losses are composed of scalable loss and non-scalable loss. For the determination of scale-up formulae in hydraulic turbines, it is important to predict the scalable loss, that is the friction losses in a runner and fixed channels, as precisely as possible. However, it is still difficult to determine them both theoretically and experimentally, partly because the boundary layer is too thin to prepare sufficient mesh size for CFD, and partly because the scalable loss cannot be separated experimentally from the measured total hydraulic loss.

Scale-up formulae have long been discussed in IEC-WG18[2] and IAHR-WG3, and also in JSME[3]. In the JSME Standard S-008 "Performance Conversion Method for Hydraulic Turbines and Pumps", the new method of theoretically derived scale-up formulae are presented[5]. In order to further improve the accuracy of scale-up, it is necessary to develop a method of more precisely predicting scalable loss.

The present study presents a prediction of scalable loss in a runner channel of arbitrary three-dimensional shape. The prediction is composed of a 3-D Euler calculation for the main flow in a runner channel and the 3-D boundary layer calculation. The latter is based on the momentum integral equation by use of an orthogonal curvilinear coordinates.

The present method is applied for five Francis runners of different specific speed, and the calculated results of scalable losses are compared with the results by conventional two-dimensional loss analysis.

\section{Boundary layer equation}

A three-dimensional boundary layer along a Francis turbine runner is analyzed taking 323

E. Cabrera et al. (eds.), Hydraulic Machinery and Cavitation, 323-332.

() 1996 Kluwer Academic Publishers. Printed in the Netherlands. 
the surface curvature into account. $Z$-axis of the absolute Cartesian coordinate $(X, Y, Z)$ is set on the rotating shaft and the rotating local relative curvilinear orthogonal coordinates $(x, y, z)$ is set on the runner wall as shown in Fig.1. Each intersection of calculated grid lines on the walls is selected as the origin of this local coordinates. For simplicity of theory, the directions of the grid lines on the wall are neglected, and the main flow

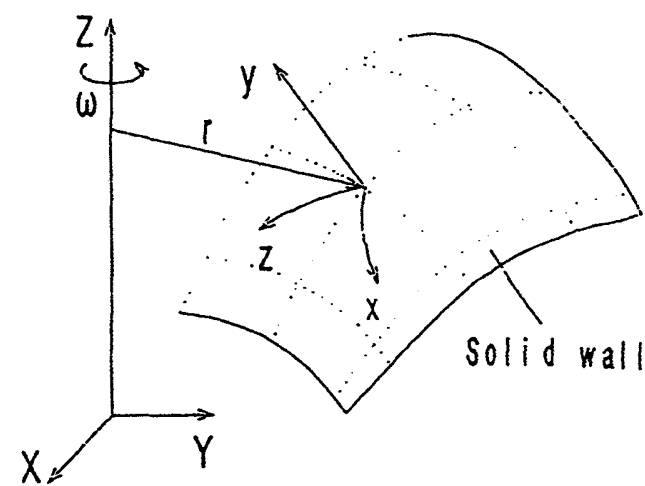

Fïgure 1. Coordinates system direction at the outside of boundary layer is taken as the $x$ direction in each origin. Moreover, the axis perpendicular to this direction on the wall is defined as $z$-axis and the normal direction to the wall is taken as $y$-axis.

The Navier-Stokes equations and the continuity equation applied to this coordinate system are given as follows.

$$
\begin{aligned}
& \frac{\partial u}{\partial t}+u \frac{\partial u}{\partial x}+v \frac{\partial u}{\partial y}+w \frac{\partial u}{\partial z}-\kappa_{z} u w+\kappa_{z} w^{2}=-\frac{1}{\rho} \frac{\partial p}{\partial x}+\nu \frac{\partial^{2} u}{\partial y^{2}}+f_{b e x}+f_{0 \circ y} \\
& \frac{\partial w}{\partial t}+u \frac{\partial w}{\partial x}+v \frac{\partial w}{\partial y}+w \frac{\partial w}{\partial z}-\kappa_{x} u w+\kappa_{z} u^{2}=-\frac{1}{\rho} \frac{\partial p}{\partial z}+\nu \frac{\partial^{2} w}{\partial z^{2}}+f_{c=z}+f_{c o z} \\
& \frac{\partial u}{\partial x}+\frac{\partial v}{\partial y}+\frac{\partial w}{\partial z}-\kappa_{x} u-\kappa_{2} w=0
\end{aligned}
$$

To stabilize the calculation, the method of calculating unsteady flow is adopted. In this method, the real length is used for the length of each axis, therefore the scale factors are fixed to unity, because the grid interval distance and the boundary layer thickness are much smaller than the radius of curvature of the axis. The velocity $\mathrm{v}$ in $y$-direction is determined by the following equation derived from Eq.(3) neglecting the last two terms.

$v=-\int_{0}^{\delta} \frac{\partial u}{\partial x} d y-\int_{0}^{\delta} \frac{\partial w}{\partial z} d y$

As shown in Fig.2, 1/n th-power law model is adopted for the velocity profile $u$ in the $x$-direction. The Marger's, shown as the followings, model is adopted for secondary flow in $z$-direction.

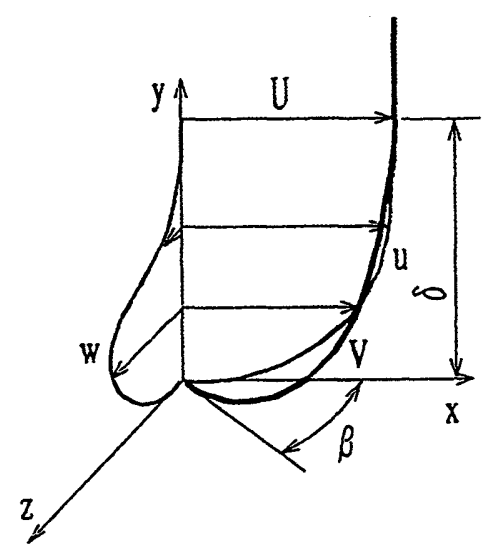

Figure 2. Velocity distribution in boundary layer

$$
\begin{aligned}
& u=U\left(\frac{y}{\delta}\right)^{\frac{1}{x}} \\
& w=u\left(1-\frac{y}{\delta}\right)^{2} \tan \beta
\end{aligned}
$$


Here, $\delta$ is the boundary layer thickness, and $\beta$ is the angle between the direction of the main flow $U$ at the outside of boundary layer and the direction of the wall stream line.

Boundary layer integral equations in $x$ and $z$ directions are obtained by substituting the above velocity distributions into Eqs. (1) and (2), and by integrating them from the wall to the edge of boundary layer under the assumption that the main flow pressure prevails into the wall surface. The pressure terms can be deleted by combining the equation of the main flow with that of boundary layer. The following equations are finally obtained for the boundary layer.

$$
\begin{array}{r}
U \frac{1}{n+1} \frac{\partial \delta}{\partial t}+\frac{\partial}{\partial x} C_{1}-U \frac{\partial}{\partial x} C_{2}+U \frac{\partial}{\partial z} C_{3}-\frac{\partial}{\partial z} C_{4}+\kappa_{2} C_{4} \\
-\kappa_{x} C_{5}=\frac{\tau_{x}}{\rho}-2 \Omega\left(\frac{\partial x}{\partial X} \frac{\partial Y}{\partial z}-\frac{\partial x}{\partial Y} \frac{\partial X}{\partial z}-\right) C_{3} \\
-\frac{\partial}{\partial t} C_{3}-\frac{\partial}{\partial x} C_{5}-\frac{\partial}{\partial z} C_{5}+\kappa_{x} C_{4}+\kappa_{2} C_{1} \\
=\frac{\tau_{z}}{\rho}+2 \Omega\left(\frac{\partial z}{\partial X} \frac{\partial Y}{\partial x}-\frac{\partial z}{\partial Y} \frac{\partial X}{\partial x}\right) C_{2}
\end{array}
$$

Here, the coefficients $C_{1} \sim C_{5}$ in the above equations are expressed as follows.

$$
\begin{aligned}
& C_{1}=\int_{0}^{\delta}\left(U^{2}-u^{2}\right) d y=\frac{2 U^{2} \delta}{n+2} \\
& C_{2}=\int_{0}^{\delta}(U-u) d y=\frac{U \delta}{n+1} \\
& C_{3}=\int_{0}^{\delta} w d y=\frac{2 U \delta n^{3} \tan \beta}{6 n^{3}+11 n^{2}+6 n+1} \\
& C_{4}=\int_{0}^{\delta} u w d y=\frac{U^{2} \delta n^{3} \tan \beta}{3 n^{3}+11 n^{2}+12 n+4} \\
& C_{5}=\int_{0}^{\delta} w^{2} d y=\frac{6 U^{2} \delta n^{5} \tan ^{2} \beta}{30 n^{5}+137 n^{4}+225 n^{3}+170 n^{2}+60 n+8}
\end{aligned}
$$

and $\kappa_{x}$ and $\kappa_{z}$ in Eqs. (6) and (7) are the curvature of the $z$-axis and $x$-axis respectively. $\Omega$ is rotating angular velocity of runner, and the last terms in Eqs. (6) and (7) show the decrease of the Corioli's force in the boundary layer.

The following experimental formula by Ludwieg-Tillman is adopted for wall shear stress $\tau_{\mathrm{x}}$ in the main flow direction.

$$
\begin{aligned}
& \tau_{x}=\rho U^{2} \times 0.123 \times 10^{-0.678\left(\frac{n+2}{x}\right)}\left(\frac{U \theta}{\nu}\right)^{-0.288} \\
& \theta=\frac{\delta n}{(n+1)(n+2)} \\
& \tau_{z}=\tau_{x} \tan \beta
\end{aligned}
$$

Equation (3) of continuity is also integrated, yielding the following equation. 


$$
\frac{\partial}{\partial x} C_{2}-U \frac{\partial \delta}{\partial x}-\frac{\partial}{\partial z} C_{3}-\kappa_{x} C_{2}+\kappa_{z} C_{3}+0.0306 U(H e-3)^{-0.653}=0
$$

Here, $\mathrm{He}$ is the entrainment of the main flow and the following equation by Standen is assumed.

$$
H_{c}=\frac{n \delta}{(n+1) \theta}
$$

After all, the variables to be determined by use of the numerical method are $\delta, n$ and $\tan \beta$ in Eqs. (6), (7) and (11).

\section{Finite difference method}

The main flow velocity $U$ at each intersection of curvilinear grid lines, shown in Fig.3, on the wall is given by the main flow calculation. In the present study, the main flow velocity was determined by solving the Euler's equation by use of a finite difference method.

As for the boundary layer calculation, Eqs.(6),(7) and (11) are converted into the

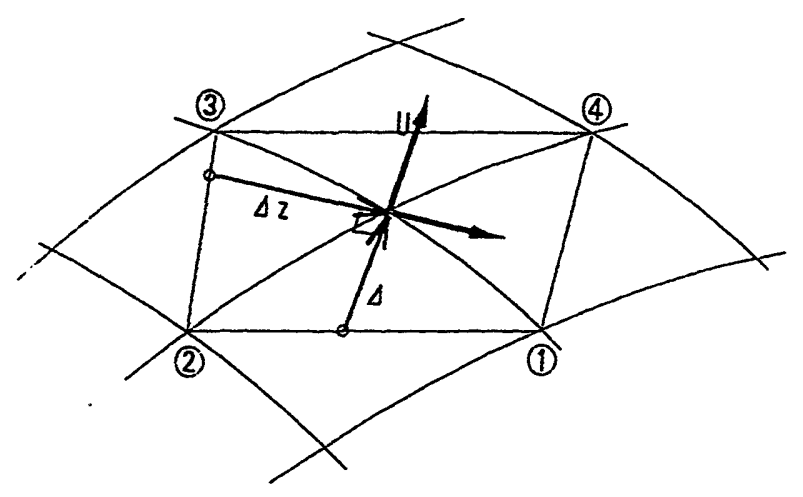

Fig.3 Local upstream FDM finite difference equations under the local coordinates system. The upstream difference scheme is used to stabilize the calculation. The upstream reference point used for FDM is set on the intersection between the lines connecting four grid points (1) (4) and the main flow line passing the origin. Each variable at the upstream reference point is determined by interpolation from the values of two grid points (1) and (2). And also, the reference point in the direction perpendicular to the main flow is similarly determined for the secondary flow. The implicit method of CrankNicolson is used for unsteady flow calculation.

For the inlet boundary condition, very small values of $\delta, n=5$ and $\tan \beta=0$ are given. The same values are also given to the whole area as the initial condition of the unsteady flow calculation. It is generally difficult to determine the flow characteristics near the corner. In the present study the equations on each wall surface is solved separately under the assumption of $\tan \beta=0$ at the comer boundary.

The solutions at each time step are determined by applying an iterative method to these equations, and the final steady solutions can be determined when the unsteady solution does not change any more.

\section{Application to Francis Turbine Runner and Main Flow Characteristics}

The preceding calculation is applied to five runners of model Francis turbines. The design parameters of these models are shown in Table 1 . The turbine $E$ is well known 
Pressure surface

$r-z$ plane
Crown surface $r$ - plane
Suction surface

$r-z$ plane
Band surface

- $z$ plane

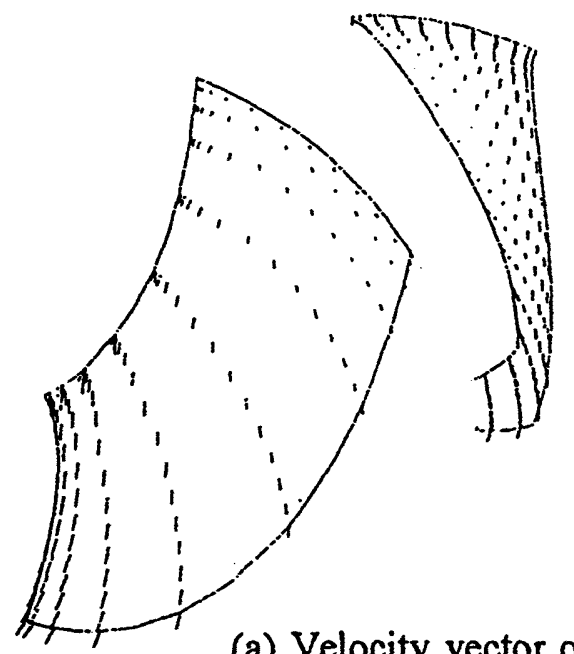

(a) Velocity vector of main flow

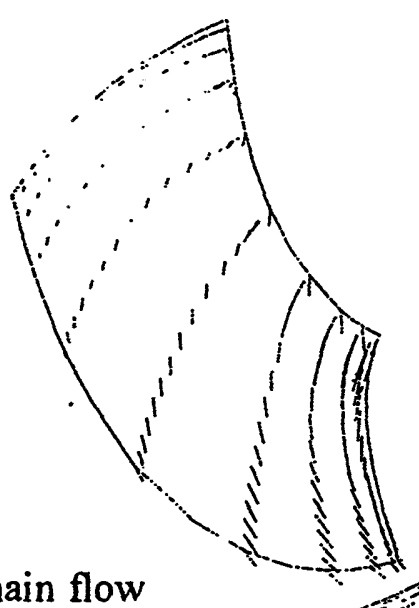

$\left|U_{2}\right|$
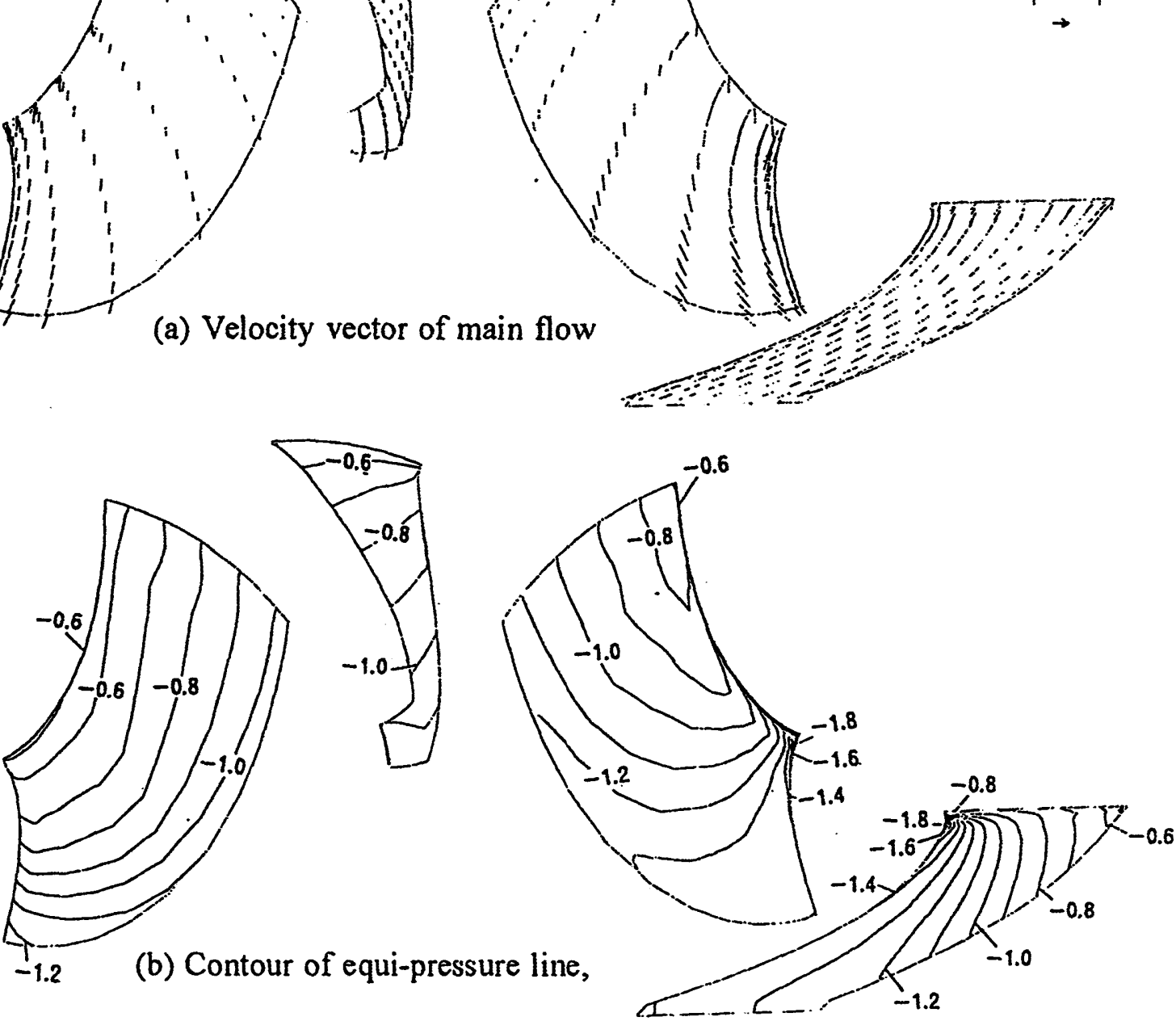

(b) Contour of equi-pressure line,
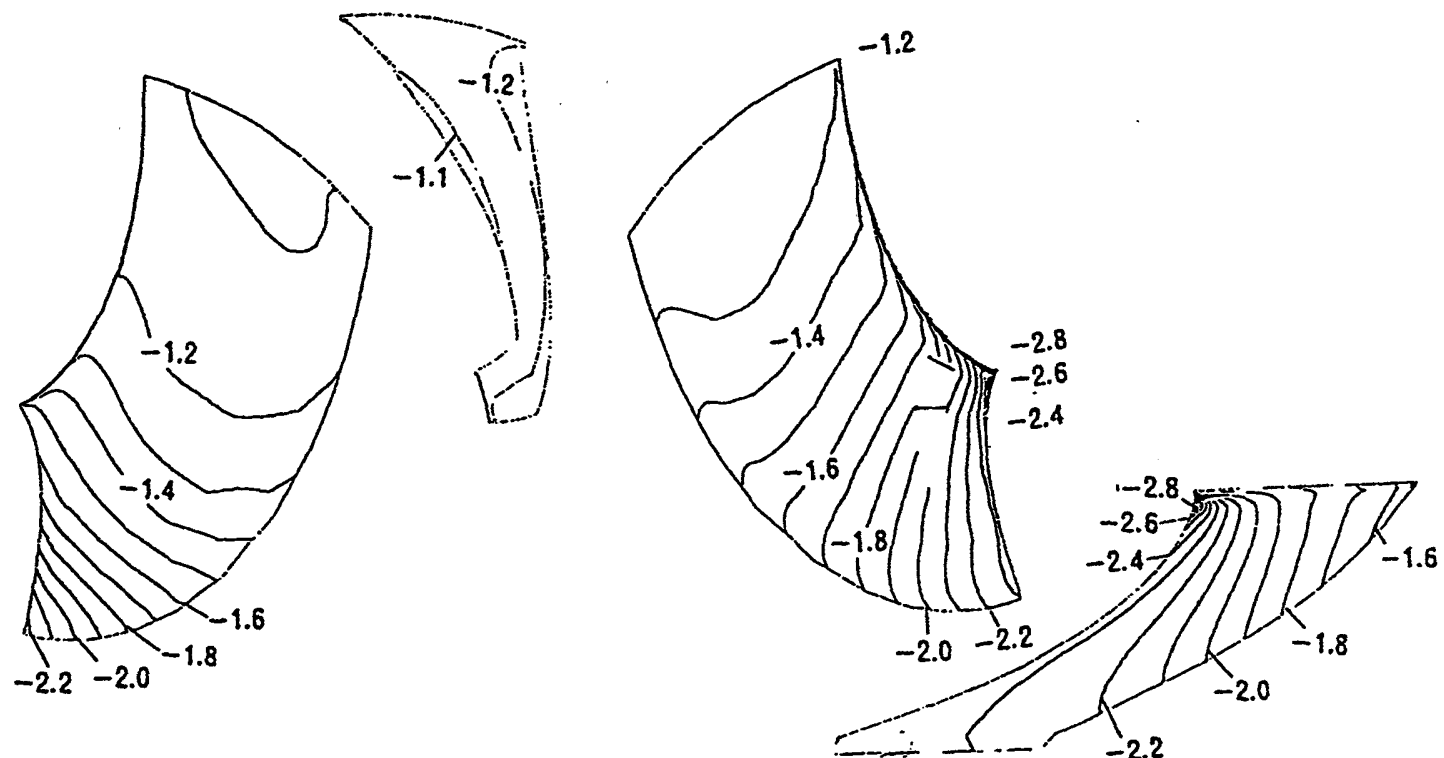

$-2.2$

(c) Equi pressure line when pressure rize due to cetrifugal force is neglected

Figure 4. Main flow characteristics (Runner E) 
Table 1. Parameters of model Francis turbines

\begin{tabular}{|l|c|c|c|c|c|c|}
\hline Runner & $\begin{array}{c}R_{\mathrm{e}}= \\
\mathrm{D}_{2} U_{2} \\
v\end{array}$ & $\begin{array}{c}D_{2} \\
(\mathrm{~mm})\end{array}$ & $\begin{array}{c}n \text { se } \\
(\mathrm{rpm}, \mathrm{m}, \\
\left.\mathrm{m}^{3} / \mathrm{s}\right)\end{array}$ & $\begin{array}{l}\text { No. of } \\
\text { runner } \\
\text { vanes }\end{array}$ & Head & $\begin{array}{l}\text { Main } \\
\text { Flow } \\
\text { Calc. }\end{array}$ \\
\hline $\mathrm{A}$ & $5.2 \times 10^{5}$ & 350 & 31 & 17 & 45 & FEM-P \\
\hline $\mathrm{B}$ & $7.1 \times 10^{5}$ & 319 & 46 & 18 & 50 & FDM-E \\
\hline $\mathrm{C}$ & $8.2 \times 10^{6}$ & 410 & 55 & 14 & 35 & FDM-E \\
\hline $\mathrm{D}$ & $6.3 \times 10^{6}$ & 350 & 75 & 11 & 20 & FDM-E \\
\hline E(GAMM) & $4.2 \times 10^{6}$ & 400 & 80 & 13 & 6 & FDM-E \\
\hline
\end{tabular}

$\mathrm{P}:$ Potential Flow, E : Euler

as GAMM Runner.

The boundary layer characteristics are analyzed only at the maximum efficiency point.

In order to show the main flow characteristics, the results of the runner $E$, as an example, are illustrated in Fig.4. These results are obtained by numerical calculation of a three-dimensional Euler's equation under the assumption of wall slip condition. The calculated velocity vector of the main flow is shown in Fig.4(a) and the contour of equi-pressure lines are shown in Fig.4(b). Here, $U_{2}$ is the peripheral velocity of the runner at reference diameter. Four surfaces of a runner channel are developed and are projected from the inside of the channel. The main flow velocity $U$ at the edge of boundary layer is used as the input data of the following boundary calculation.

The calculated results of the main flow in the runner exit section is compared with the measured data in Fig. 5 for the case of runner $E$. The horizontal axis is the distance from the outer wall of draft tube. The numerical results are averaged over the vane-to-vane channel. The calculated results agree well with the experimental results.

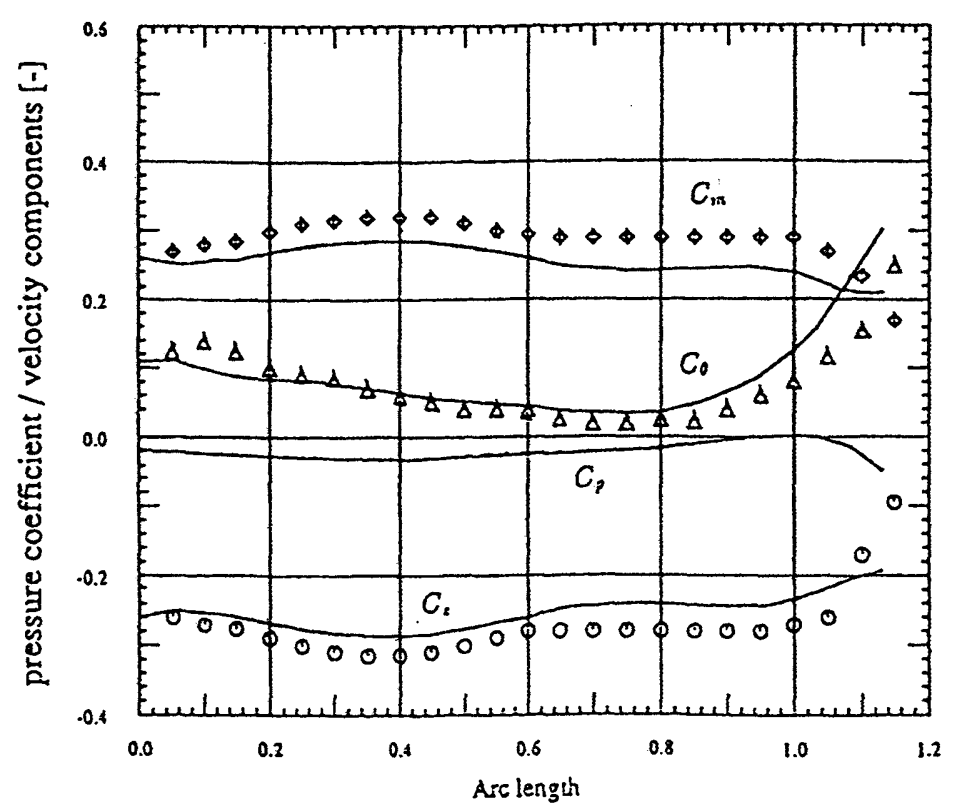

$\left(C_{\rho}:\right.$ Pressure, $C_{z}$ :axial velocity, $C_{*}$ :Tangential velocity, $C_{\mathrm{m}}:$ meridional velocity)

Figure 5. Outlet Flow characteristice in runner $E$ and comparison with measurments 


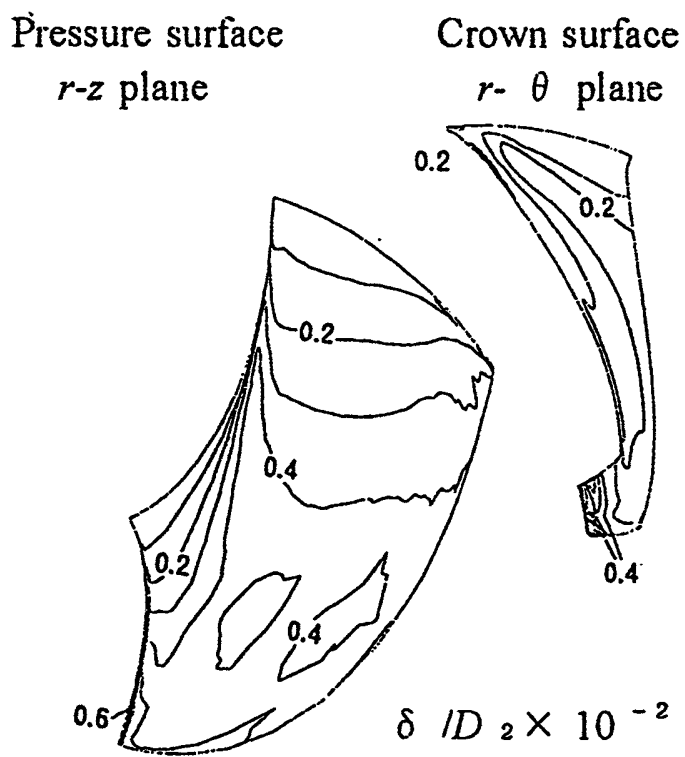

(a) Boundary layer thicknes,
Suction surface

$r-z$ plane
Band surface

$\theta$-z plane
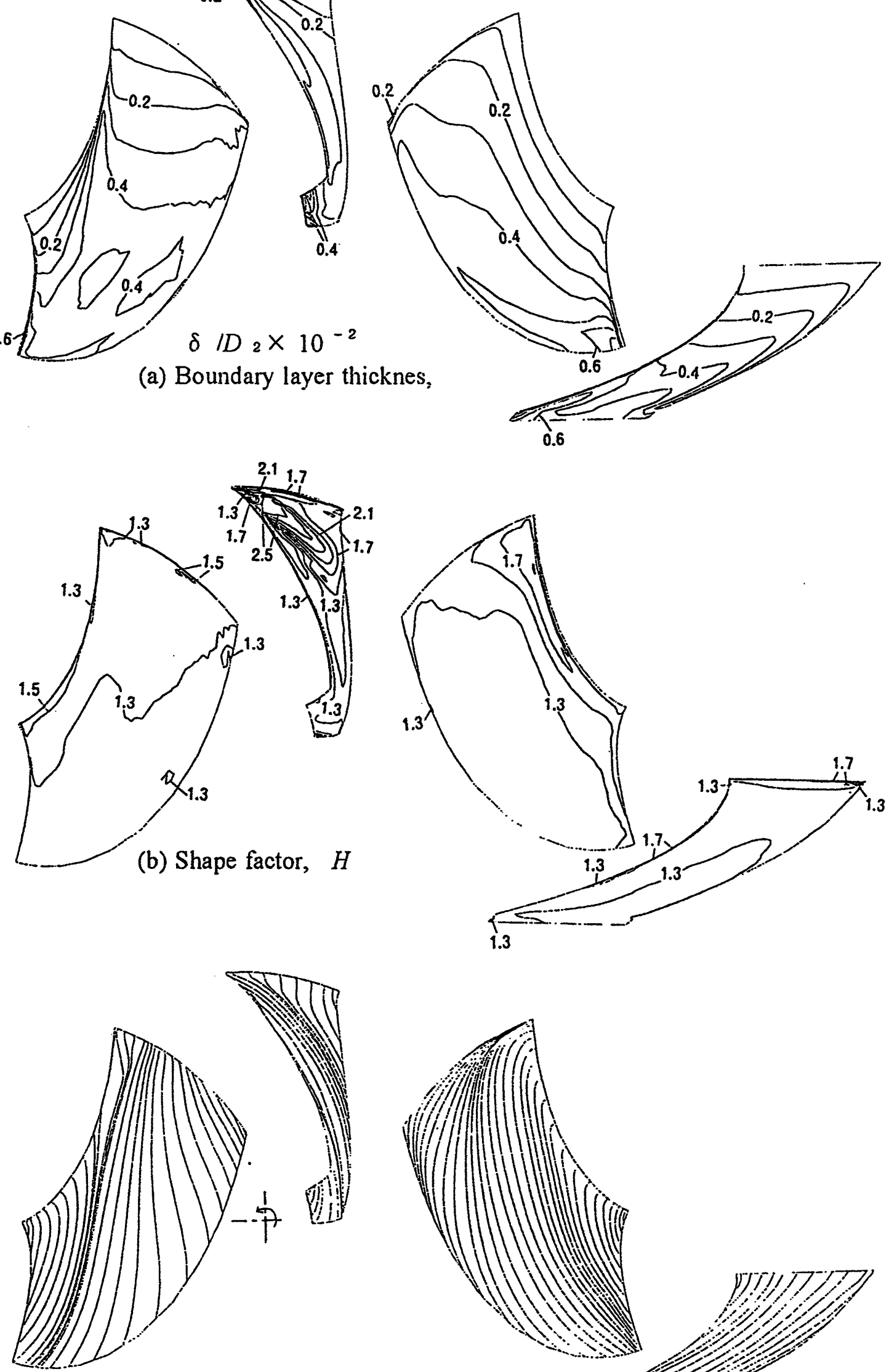

(c) Wall stream line

Figure 6. Results from 3-D boundary layer calculation (Runner E) 


\section{Results and Discussions of Boundary Layer Calculation}

To discuss the boundary layer characteristics, the contours of the equal boundary layer thickness $\delta$, of the equal shape factors $H$, and the wall stream lines are illustrated in Fig.6 for the case of runner E. The boundary layer calculation were performed without any trouble, although reverse flow occurs near the crown wall of the pressure side in the main flow.

On the vane suction surface and on the band surface of the boundary layer grows from the leading edge, but on the vane pressure surface it grows from the entrance of the crown surface, since the main flow enters into the pressure surface through the crown surface as shown in Fig.6(a).

The Corioli's force works strongly in the main flow where the velocity is large, and balances with both the pressure gradient and the inertia force. Because the Corioli's force works weakly near the wall where flow is slow, the wall stream line turns to the opposite direction of Corioli's force, or turns to the direction in which the real pressure decreases if the pressure rise due to centrifugal force is removed as shown in Fig.4(c).

On the crown surface shown in Fig.6(b), the shape factor becomes very large near the corner between the inlet and the pressure surface where flow velocity is slow, and the boundary layer fluid is transferred along the wall stream line which deviated from the pressure side to the suction side between vanes. This flow behavior is mainly caused by the pressure gradient from the pressure side to the suction side rather than the Corioli's force.

In the suction surface of the vane, the boundary layer gradually grows toward the downstream near the leading edge, but the stream lines turns to the band side with an increase in the boundary layer thickness. This is because the pressure decreases from the crown side to the band side due to the meridian curvature of channel.

Using the present method, the hydraulic energy loss due to friction can be predicted by use of energy thickness of the boundary layer. The friction loss on the wall can be estimated as a energy defect at the channel exit. The energy defect is calculated from the energy thickness $\theta *$ at the exit surface for the case of three dimensional boundary layer.

$$
\theta^{*}=\frac{1}{U^{3}} \int_{0}^{0} V^{\prime}\left(U^{2}-V^{2}\right) d y
$$

Here, $V$ is the velocity component perpendicular to $V$, which is velocity in the boundary layer(Fig.2), on the exit section.

On the other hand, in the performance conversion, the ratio $\delta \mathrm{Er}$ of friction head to effective head is very convenient for standardization, as adopted in IEC Code or JSME Code. The ratio $\delta_{\mathrm{Er}}$ is obtained by the following equation.

$$
\delta_{\mathrm{Er}}=\oint\left(U^{3} \theta^{*}\right) d l / g Q H
$$



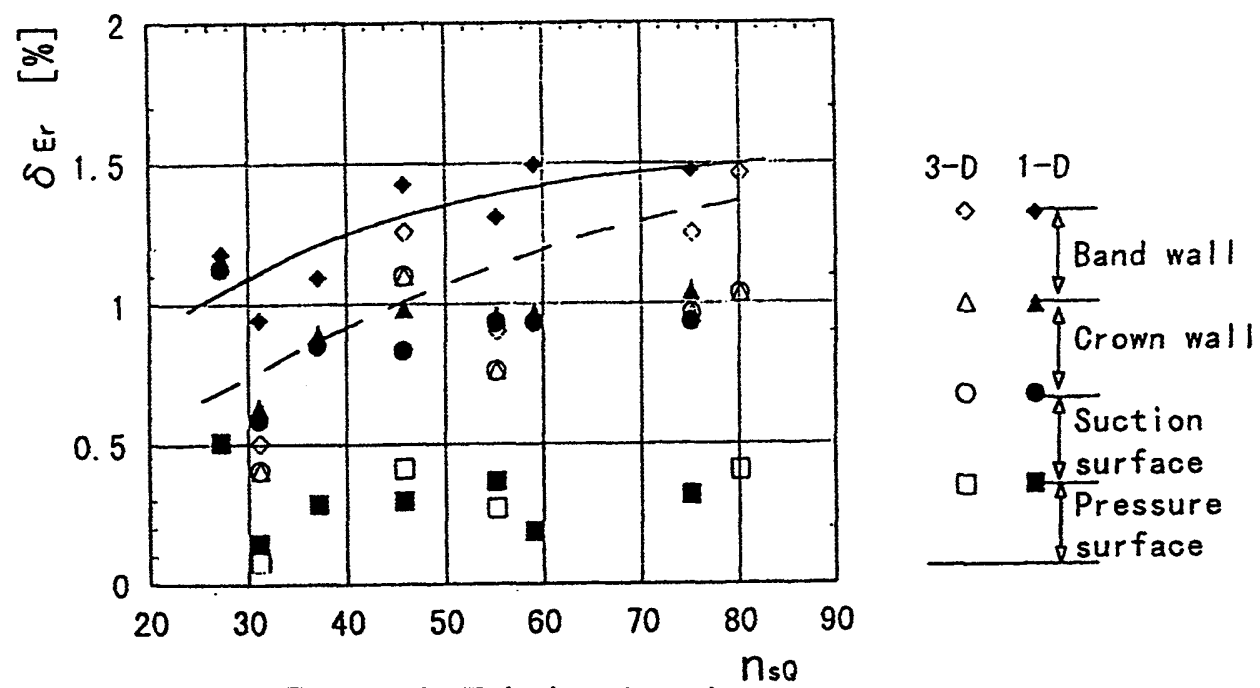

Figure 7. Friction loss in runner
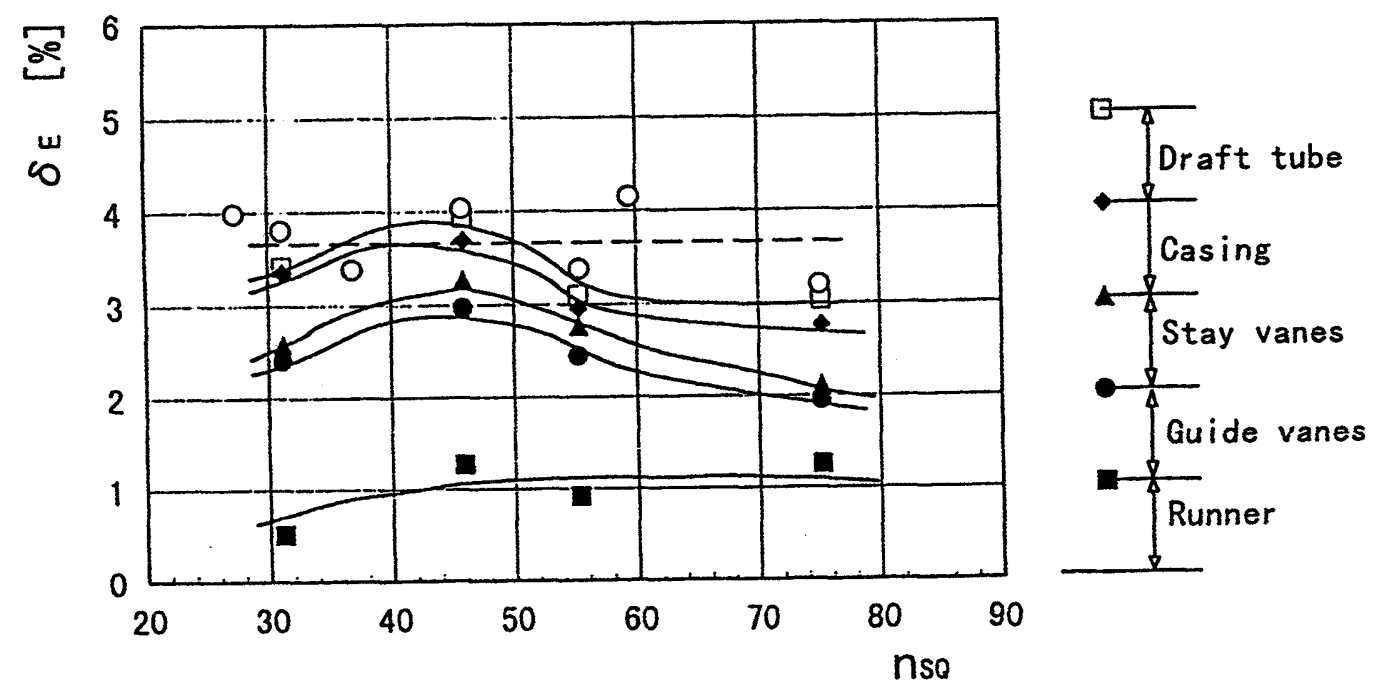

Figure 8. Friction loss of turbine

Here, $l$ is the distance along the contour of the exit wall. The curvilinear integration concerning $l$ is performed along the exit wall edge of all channels of the runner.

The present calculation was applied to all turbine runners A-E and the calculated friction loss ratio $\delta_{\mathrm{Er}}$ are plotted against specific speed $n s Q$ Fig. 7. The notations $\bigcirc \diamond \square \triangle$ are the results from the present calculation, and the notation $\bigcirc \mathbf{\Delta}$ are those of the conventional two dimensional analysis, in which the flow is assumed to be along the grid line, and the energy equation and Rotta's equation are used.

The total friction loss in a runner becomes larger with an increase in the specific speed, but the scatter of the data is large because of the difference in the design philosophy and manufacturing tolerance of each turbine. The solid line indicates total friction loss obtained by two dimensional analysis.

Figure. 7 shows that the total friction loss from two dimensional analysis becomes larger than that from the present analysis. Due to the secondary flow, the boundary layer thickness must be thinner in the thee-dimensional analysis than in the two dimensional analysis, because the fluid in the boundary layer is removed by the secondary flow in the 3 Danalysis. 
Figure. 7 also reveals that the friction loss on the vane occupies large part of the total friction loss of a runner. On the other hand, on the crown surface, the main flow velocity is slow and the wall stream line leans. Therefore the boundary layer thickness in the exit section is thin and the friction loss becomes thus very small.

The friction loss ratio $\delta_{\mathrm{E}}$ of the casing, stay vanes, guide vanes, and draft tube of turbines from A to D are shown in Fig.8. All the results except for loss of the runner are obtained by two dimensional calculation. Figure. 8 reveals that the friction loss on guide vanes becomes smaller with an increase in specific speed. The notation $O$ shows the sum of friction losses on all surfaces obtained by using two dimensional analysis. The dotted line is the averaged total scalable loss and is seen to be almost independent of the specific speed, and $\delta_{E}$ takes the value around 0.037 .

\section{Conclusions}

The calculation method of three dimensional boundary layer developed by use of upstream FDM with local curvilinear coordinate system, is applied to five types of Francis turbines. The following conclusions are obtained.

(1) The present method can be solved without trouble, even if complicated flow including reverse flow is induced such as the flow in the pressure surface.

(2) The present method is able to estimate the influence of the secondary flow, which is perpendicular to the main flow. The friction loss obtained by this method becomes smaller than that obtained by two dimensional method.

(3) The averaged total friction loss ratio on all surfaces obtained by using two dimensional analysis is about 0.037 in Francis turbine, and it is almost independent of the specific speed.

\section{Acknowledgements}

Calculation was performed as a part of the activity of ISME WG "Performance Conversion Method for Hydraulic Turbines and Pumps". The main flow data for 3D Euler, 3D potential flow and 2D boundary layer data were offered by the committee members, Dr. Nagafuji and Mr. Suzuki in Toshiba Co.Ltd., Mr. Miura in Hitachi Co.Ltd. and Mr. Miyagawa in Mitsubishi Heavy Industry Co.Ltd.. The authors would like to express sincere gratitude to the WG members.

\section{References}

1. Arakawa, C., Samejima, M., et al., A 3D Euler solution of Francis runner using pseudocompressibility, 3D-Computation of Incompressible Internal Flows (NNFM39), (1989), 65-69

2. IEC draft code 4(CO)47-1987. Determination of the prototype performance from model tests of hydraulic machines with consideration of scale effects

3. ISME S008(1989), Performance conversion method for hydraulic turbines and pumps

4. Suzuki, R., An assessment of the loss distribution in Francis Turbines, Proc. of 18th IAHR Sympo., (1996)

5. Ida T., et al., Recent development of studies on scale effect in Japan, Proc. of 18th IAHR Sympo.. 